\title{
Covid-19 Information on YouTube in the Early Pandemic
}

\author{
John C. Paolillo \\ Indiana University \\ paolillo@iu.edu
}

\author{
Brian P. Harper \\ Indiana University \\ bpharper@iu.edu
}

\author{
David Axelrod \\ Indiana University \\ daaxelro@iu.edu
}

\begin{abstract}
As people sheltered globally during the COVID-19 pandemic, many YouTube videos and channels pivoted to providing COVID-19 information. But were these videos helpful and constructive or did they undermine official public health messaging? This paper addresses these questions through a mixed methods study of COVID-19 videos on YouTube produced from January to May 2020. We find that a preponderance of YouTube COVID-19 videos either came from major news studio outlets or offered official public health communication. While YouTube moved quickly against obvious false messages, other more subtle ones still managed to leak through. Medical information channels presented conflicting information at times, reflecting factors such as medical uncertainties, political currents, and audience pressures associated with uncertain information around a novel pandemic.
\end{abstract}

\section{Introduction}

Changes in people's dominant social experiences are readily felt in social media, and the spread of awareness about the COVID-19 pandemic in early 2020 was no exception. From the earliest reports of a mysterious pneumonia emerging in China, to mass infections on cruise ships, to chaotic scenes of overwhelmed hospitals in Italy, New York and other locales, to lockdowns and travel bans imposed by institutions and governments straining to cope, all phases of the pandemic were evident on social media. The public posted, linked to media stories, commented on, experienced and documented the looming and then painfully present threat. This contributed to what the WHO decried as an "infodemic", largely blaming social media misinformation [1], becoming a point of debate in scholarly literature. Though some researchers initially critiqued the WHO's framing [2], studies such as [3], found correlations of social media use with lower compliance to health precautions, and broadcast media use with better compliance. YouTube specifically received criticism on account of its long, problematic record with respect to the dissemination of medical and public health information [4].

One of the larger social media platforms, YouTube was an important hub of COVID-19 information, ranging from the identification of the disease and its symptoms, to the location and spread of infections, to measures for hand-washing, sanitation, and mitigation of disease spread, to government public health communication, etc. YouTube also served as one of the early vehicles of false cures (e.g., Miracle Mineral Solution, "MMS") and conspiratorial origin stories (e.g., the story that 5G wireless causes COVID-19). What then was the state of COVID-19 medical information on YouTube during the early pandemic, and to what extent or in what ways did it contribute to confusion about COVID-19 information? What sort of COVID-19 information could a viewer expect to encounter during this period, and how did it evolve as the pandemic progressed?

Like other social media platforms, YouTube immediately attempted to mitigate false information about COVID-19. Videos promoting quack therapies such as MMS were deleted as far back as January 2020, and 5G-coronavirus videos were deleted nearly as quickly as they were posted. These measures were not completely effective. For example, although rapidly deleted from any channel that posted it, the misleading documentary Plandemic remains available contained within numerous commentary or reaction videos. Regardless, YouTube's measures were strict enough that they even affected some of the larger channels promoting mainstream medical information, while even very basic information about the virus remained hotly debated within the global medical community, e.g., the nature of its infective spread (via droplets, surfaces or aerosols), and the viability of treatment options (ASIDs, steroids, novel antiviral drugs, chloroquine, etc.). Pandemic-related medical information could burnish or mar any nation's global image with respect to rivals, as in the case of China and the United States' contentious relationship. These circumstances confounded the public, governments, and social media platforms in discerning which COVID-19 information should be seen as correct.

\section{Background}

Analysis of any content on YouTube faces certain common challenges. One problem is scale. While many studies apply quantitative or network approaches $[5,6$, 7], this entails less attention to specific messages and more superficial knowledge about what the platform 
contains. Other approaches focus on specific genres [8], thereby narrowing the scale and scope. Both types of approach tend to treat observations as synchronic (an exception being [9]), whereas YouTube is always changing. Not only do videos and channels come and go, YouTube's promotion and recommendation of videos also constantly changes, amplifying the reach of some content [10], but making other content invisible. YouTube is also claimed to have a participatory culture [11] in which one can expect information from a wide variety of sources varying greatly in quality. Some health information, for example, may center upon alternative medicine or fashion-centric diet advice. In the fluid world of COVID-19 information, these issues are important.

Prior to and independent of the pandemic, YouTube's role in negative social trends was already under scrutiny. Studies have debated toxicity in the platform's video comments [12] and the platform's role in political radicalization, for example. Ribeiro [13] suggests that overlapping commenter communities induce a migration toward more radical content, whereas Ledwich et al. [14] argue that YouTube's recommendations actually push users toward more mainstream and left-leaning content. Faddoul et al. [15] suggest that YouTube has been effective in slowing the spread of inaccurate content: taking into account views per video, their study found that in spite of a boom in conspiracy theories on YouTube at the end of 2018, they declined beginning in April 2019 to a nadir in May/June 2019. While such observations might be regarded as encouraging, producers of marginal content often employ strategies to avoid detection and may operate in gray areas that receive less scrutiny. Such strategies for skirting moderation can play an important role in an evolving information landscape like that of the COVID19 pandemic.

YouTube has also been scrutinized with respect to health/medical information and misinformation. Such studies often employ instruments designed for nonvideo forms of medical information on modest-size samples. For example, Briones et al. [16] use the Health Belief Model to analyze 172 videos that contain attitudes to preventative procedures for HPV. The sample consists of the union of the first 50 query results (accessed November 1, 2010) for each item in a list of HPV search phrases, subtracting any duplicates. The study assessed the arguments within the videos for their valence toward the vaccine as well as the source of authority for the arguments. A substantial portion of the videos examined, $28.5 \%$, falsely suggested vaccines were ineffective for HPV, 36\% mentioned a psychological risk from the vaccine and 53.5\% claimed a high physical risk.

An even smaller study on HPV vaccine YouTube videos [17] analyzed 35 videos and each video's first
100 comments. Six search phrases were queried from June 22, 2014 to December 19, 2014 and each query's top 10 unique results were included in the study sample. This study found $57 \%$ of their sample to be antivaccination videos compared with $31 \%$ that were provaccination. In addition, pro-vaccination content was much more likely to report information about HPV accurately and include key facts about HPV. The prevalence of anti-vaccination narratives around a wellresearched topic is noteworthy given the likelihood that the developing nature of the COVID-19 pandemic facilitated scientifically flawed narratives.

Another tool, DISCERN, has been used to study YouTube videos on misinformation around prostate cancer [18]. DISCERN is a short questionnaire that has been developed into a standardised index for quality of consumer health information. The questions used in DISCERN address a source's overall reliability and specifically, the quality of information on treatment choices. A high cumulative rating indicates a highquality source. One study about the COVID-19 pandemic [19] used DISCERN to analyze videos uploaded by physicians, hospitals, health organizations, educational channels, and news organizations. The sample used by Szmuda et al. [19] was put together using seven COVID-19 related search phrases queried on March 12, 2020 and further reduced to a sample of 137 videos after filtering out duplicates, non-English videos, off-topic content, and videos exceeding an hour duration. Szmuda et al. found that all sources uploaded videos of fair or poor quality according to the DISCERN evaluation. Videos uploaded by news organizations, constituting nearly half of the videos in their corpus, had the worst DISCERN scores along with hospital channels. Although this suggests widespread exposure to low-quality information on COVID-19, one study [20] found that less than $2 \%$ of the YouTube videos they examined propagated unsubstantiated claims about the pandemic and that most content about COVID-19 was produced by news outlets. However, low quality videos received significantly more engagement than videos from reputable sources and this paper highlights the dangers of vital medical information being shared on a social media platform like YouTube which provides financial incentives for attention-seeking content.

\section{Methods}

Our research method adopts as one component a content analysis approach employing grounded theory. This approach allows us to accommodate the diversity of COVID-19 information, as it accommodates messages that cannot be known a priori. We also need to evaluate the propensity of different messages, requiring a quantitative analysis. We accomplish this by collecting metadata for COVID-19 videos, developing 


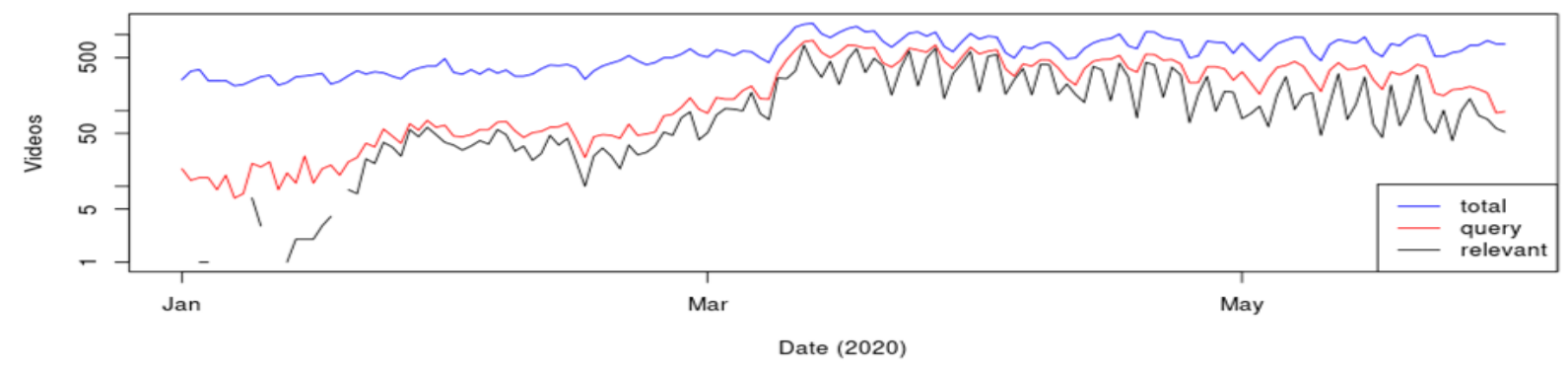

Figure 1. Timeline of videos collected, queried, and then culled for relevance.

and applying the content analysis coding, and conducting a quantitative analysis via Principal Components Analysis (PCA) and cluster analysis. Our collection, querying and culling of videos to create the sample and coding observations forced us to intensively study and take notes on this content in a manner also well-suited to qualitative analysis.

Video metadata for COVID-19 videos was collected using a custom Firefox browser add-on that extracted video IDs from YouTube pages and forwarded them to a server that collected their corresponding metadata into a database using the YouTube Public Data API. This permitted metadata collection during ordinary browsing, obtaining video and channel metadata from a YouTube video's page and video metadata for any recommended videos. The add-on also provided direct feedback on the YouTube page being browsed as to which videos were previously in our database and which had only just been discovered. Daily sessions of intensive searching and browsing COVID-19 topics began in January 2020, focused on finding as many COVID-19 videos as possible, irrespective of source country (for language reasons, English predominates in our sample, but French, Italian, Spanish, Hindi, and Tagalog videos and channels were frequently visited). The database generated reports as web pages with links to the videos and channels collected, so that we could revisit them for new video recommendations not previously discovered. YouTube feeds recommendations partly based on matching publication dates, so this practice allowed us to fill gaps in the timeline. Emphasis was placed on finding videos published within the January through May period, but earlier and later videos were not excluded from browsing, if these looked to contain material relevant to the pandemic, candidate treatment options, etc. We selected May 31 as a stopping date for our analysis period after it became clear that the murder of AfricanAmerican George Floyd by the white Minneapolis policeman Derek Chauvin on May 25, 2020 (and subsequent protests against police racism) produced a discontinuity in the COVID-19 topic on YouTube. Data collection to fill in gaps in our January-May record continued until October, 2020, when our API access was arbitrarily revoked without notice, thereby necessitating revamping of our data collection apparatus.

Video titles and descriptions were searched using a disjunction of several COVID-19 terms to nominate a candidate set of 37,950 videos for possible analysis. These terms are: adhanom, antibody, antigen, bioweapon, cdc, covid, corona, coronavirus, cough, coup, crash, crisis, cure, disease, disinfect, epidemic, immune, infect, influenza, flu, mask, mers, mutation, pandemic, pneumonia, quarantine, sars, tedros, transmit, treatment, vaccine, virus, wenliang, and wuhan. Additional search terms had little to no effect on the candidate set size.

The candidate set was then manually culled for relevance to the treatment of COVID-19; we excluded videos solely addressing political, economic, and human-interest stories not directly connected to medical treatment. This gave a final culled set of 23,169 videos. Figure 1 presents a timeline of the counts of each of these three stages of sampling: videos for which our browsing and searching gave us metadata, videos that were selected by our keyword search, and videos remaining after our manual culling. The culled set was then randomly sub-sampled for content analysis coding.

We chose the statement as our coding unit: since videos vary in length, they may contain multiple statements about COVID-19, its origins and treatment. Coding statements allowed us to be adaptive to the novel content found in our sample and gain more detail from the relationships between our codes. Statements may be syntactically mono-clausal or multi-clausal, although we avoided meanings that were merely implied. We recognized two types of statements of interest: disease origin statements and treatment statements. For each type, we recognized four semantic slots and developed a set of categories for each via discussion among all three co-authors. Origin statements had slots for cause (for what gave rise to the pandemic) and benefactor (for who was claimed to benefit); treatment statements had slots for treatment (self-evident) and framework (medical or other system of knowledge under which the statement was asserted). Both types of statement had 
slots for valence (positive, negative, etc.) and authority (the source of authority for the statement). While we tested more codes and allowed coders to input their own novel codes, we settled on 70 which were considered for a total of 764 statements in 364 videos, ranging from one to fourteen statements per video (Table 1). As our sample largely consisted of English language content, codes for Donald Trump and Anthony Fauci were used often enough that they were left alone rather than be consolidated like the codes used for other leaders.

Table 1. Codes used in each of the four statement slots in the video analysis.

\begin{tabular}{|l|l|}
\hline $\mathbf{1}$ & $\begin{array}{l}\text { Cause: animaleco, bioweapon, hoax, } \\
\text { impurity, in air, lab fumble, lab made, onstuff, } \\
\text { talk/cough, wireless, any } \\
\text { Treatment: vaccine, mask, distance, } \\
\text { parcetamol, ACE inhibitor, dismantle, rituals, } \\
\text { spirit warfare, ventilator, disinfectant, travel } \\
\text { restrictions, handwashing, ultraviolet, de- } \\
\text { stressing, bleach, heat/sauna, spices, alcohol, } \\
\text { chloroquine, breathing, azithromycin, zinc, } \\
\text { testing, vitamins, health norms, vitamins, } \\
\text { remdesivir, shield, steroid, aspirinoid, surgery }\end{array}$ \\
\hline $\mathbf{2}$ & $\begin{array}{l}\text { Benefactor: US, China, Fauci, Donald } \\
\text { Trump, elites, God, Satan, none } \\
\text { Framework: pharmacological, Chinese } \\
\text { medicine, ayurveda, self-medicate, } \\
\text { homeopathy/naturopathy, economic, spiritual }\end{array}$ \\
\hline $\mathbf{3}$ & $\begin{array}{l}\text { Valence: pro, con, mixed, absolve, blame, } \\
\text { benefit, victim }\end{array}$ \\
\hline $\mathbf{4}$ & $\begin{array}{l}\text { Authority: science, MD, Fauci, Public Health } \\
\text { Institution, CDC, WHO, Donald Trump, } \\
\text { religion, politics, finance, journalistic }\end{array}$ \\
\hline
\end{tabular}

A configurable interface was constructed for both developing and using the codes (Figure 2). As can be seen there, a video is selected by its ID from a randomized list in the left-hand column; the coding status of each video being displayed in the button style. The link to the selected video is placed above the codes, which are colored by slot. Selecting a code button fills in a text field corresponding to the slot for the code, and an apply button sends the selected codes to the database. The interface permits relatively rapid coding of the videos, with most of the coder's time spent on examining the video itself.

Complex coding schemes like ours are incompatible with traditional inter-rater reliability computations. Instead, inter-rater reliability was addressed post-analysis using sanity checks (see below). The analysis itself was performed using PCA followed by Gaussian mixture model-based cluster analysis [21]. For the PCA, we began by constructing an incidence matrix of codes and code combinations within each video: the frequency of each code within each video was counted, as was the frequency of any code bigram, trigram or full statement (referred to as "code combinations", below). In this way, correlations across the different coding slots could enter the analysis. As the full number of codes and code combinations $(1,182)$ exceeds the number of videos coded (364), the resulting PCA is a Q-mode PCA [22].

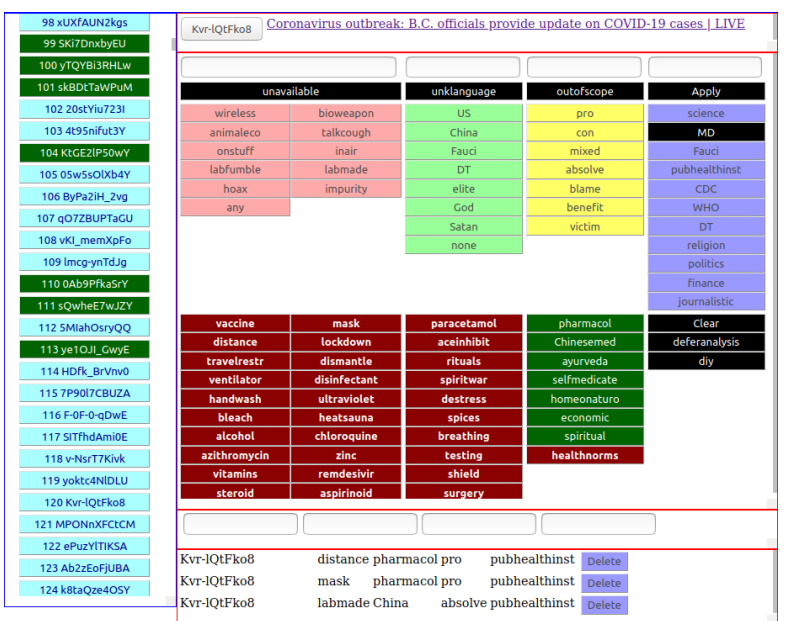

Figure 2. Screenshot of our coding interface.

Since the incidence matrix is composed of count data, we consider a multivariate Poisson model to be more suitable to the data than a standard, multivariate normal PCA. Hence, PCA was performed using the R package PLNmodels [23]. While the package allows for a joint regression-PCA model of multivariate count data, the relative inflexibility of the regression feature was unsuitable for our application. Hence, we opted for a model with only an intercept term and no offset or regression covariates. The model selection criteria differ with respect to how many dimensions to extract: the Bayesian Information Criterion (BIC) favors a 2component solution whereas the Integrated Completed Likelihood (ICL) criterion is ambiguous between 2 and 3 components. So as not to discard potentially relevant information, we settled for the three-component solution, where PC1 accounts for $84.72 \%$ of the explained variance, PC2 for $8.11 \%$ and PC3 7.17\%, with $61 \%$ of the total variance explained.

The principal components scores of the videos were submitted to Gaussian mixture model clustering using the $\mathrm{R}$ package Mclust [21, 24, 25]. Using a BIC criterion, we selected an optimal cluster solution; this was a ten-cluster model. Inspection of the members of these clusters suggested that they were reasonably interpretable, and so we proceeded with this solution for our final interpretation. A similar attempt of clustering 
the code combinations was also made but we found these not to produce satisfactory cluster solutions because of convergence problems. In addition, the large number of columns in the Q-mode incidence matrix yields many variables with low weightings, and consequent large clusters that are not readily interpretable. For these reasons, we based our interpretation entirely on the video clusters.

Two sanity checks were performed prior to the interpretation of clusters. For one, we cross-tabulated the clusters by individuals coding the videos; this is given as Table 2, inspection of which suggests that although one coder coded more videos than the other two, no coder-cluster association is observed. Furthermore, using the chi-squared likelihood ratio test for log-linear models, we can formally reject the hypothesis of coder-cluster association: neither the model comparison chi-square of 6.847 (18 df) nor the residual values for individual cells (the largest of which is 0.36 at $1 \mathrm{df}$ ) are significant at the $\mathrm{p}<0.05$ level. Hence, inter-coder differences are not responsible for our observed clusters. Similarly, we plotted the locations of videos on each principal component by video publication date and colored by cluster (omitted for space reasons). The only pattern emerging in these plots was a banded structure, indicating that the PCs do indeed separate the clusters but are uncorrelated with time. For some clusters, parts of the band corresponding to January and February were missing, though this is because these two months had the fewest videos in our sample representing them, on account of less COVID19 activity on YouTube at that time.

Table 2. Number of videos in each of ten clusters for the three coders.

\begin{tabular}{l|llllllllll} 
& $\mathbf{1}$ & $\mathbf{2}$ & $\mathbf{3}$ & $\mathbf{4}$ & $\mathbf{5}$ & $\mathbf{6}$ & $\mathbf{7}$ & $\mathbf{8}$ & $\mathbf{9}$ & $\mathbf{1 0}$ \\
\hline $\boldsymbol{A}$ & 29 & 13 & 56 & 23 & 13 & 15 & 17 & 19 & 8 & 17 \\
$\boldsymbol{B}$ & 8 & 7 & 15 & 7 & 5 & 4 & 5 & 9 & 4 & 7 \\
$\boldsymbol{C}$ & 7 & 9 & 23 & 9 & 5 & 7 & 6 & 6 & 4 & 7
\end{tabular}

\section{Results}

\subsection{Clusters and descriptions}

While all three principal components contribute to the separation of the clusters, of the three, only the first admits a direct interpretation. Figure 3 presents a plot of videos on PC1 and PC2 (the two components with the greatest variation) and colored by cluster; about half of the clusters concentrate on the left end with the others spread out on the right. The clusters on the left represent more news-like content with more conventional
COVID-19 messages, while the diffuse clusters represent YouTube-specific genres with less conventional messaging.

While the clusters on the left of Figure 3 are similar in being more news-like, their topic matter varies by cluster. The yellow-green cluster (\#3 in Table 2, indicated in Figure 3 with ) on the left is the largest and perhaps the most characteristic of our sample. It consists of new updates about the pandemic from broadcast journalists, government officials, as well as YouTube-based news creators. These videos usually contain morbidity and mortality statistics, policy updates about social distancing and travel restrictions, and reiteration of standard public health guidance of the time: instructions to socially distance, quarantine if infected, and handwash are constant, while guidance regarding mask-wearing varies over time. Though then New York governor Andrew Cuomo's updates are perhaps the most memorable US examples, these sorts of press conferences took place in many countries and at many levels of government, sometimes streamed directly to YouTube.

The red cluster (\#1 ) in the lower left consists of doctors or other medical professionals explaining details about COVID-19, often in interviews with journalists. Unlike the yellow-green cluster which usually relied upon the authority of public health institutions like the WHO, CDC, and national health ministries, here the interviewee's authority as a doctor, possibly from a prestigious institution (e.g., Johns Hopkins), was salient. Doctors were generally quite consistent in their basic public health instructions (handwashing and social distancing), although details about the pandemic vary depending on when the video was produced. Some of these videos were produced by the doctors themselves rather than as part of news programs. Most were intended for the general public with the level of detail calibrated for a lay audience, although some were more complex, especially ones produced by doctors themselves, and offered more nuanced treatments of the efficacy of policies like lockdowns.

The orange cluster (\#2 ), also in the lower left, contains more political framings of the pandemic concerned less with medical/public health information. Members of this cluster include discussions and criticisms of President Trump's press conferences, arguments about the economic cost of lockdowns and social distancing, and interpretations of how the pandemic alters the fortunes of various politicians. Videos discussing the pandemic from a religious perspective were also represented in this cluster, focusing on how to live religiously in the context of the pandemic and the many changes to life imposed by lockdowns and social distancing. During most of our sample frame (January-May), social distancing and lockdown requirements were not yet strongly 
politicized, and misinformation regarding them was not yet rampant; this may explain the proximity of the orange (political and religious) and red (medical information from doctors) clusters. Both clusters are news-like, but focus on different sources of authority, such as individual doctors, pundits, or religious figures, rather than public health institutions.

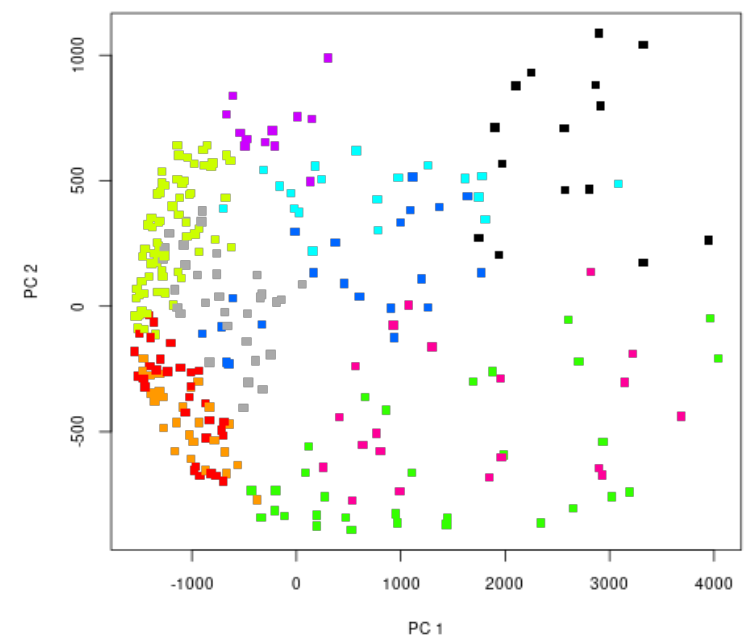

Figure 3. Cluster graph representing principal components 1 and 2.

The gray cluster (\#8 ) on the left consists of videos explaining more medically complex topics like the evidence for novel treatments and how COVID-19 testing works. As is characteristic of the clusters on the left-hand side of the graph, many of the videos here are from broadcast news providers, though not exclusively. Videos from doctor/YouTubers were also represented in this cluster discussing drugs like hydroxychloroquine, remdesivir, and ivermectin, among others. Although some of these, especially hydroxychloroquine, became central to treatment misinformation over the Summer, the videos in this cluster could best be described as 'cautiously optimistic' about the drugs based on the limited research available at the time. The difficulties of testing for COVID-19 were also discussed in these videos, while acknowledging the need to do so. The last news-like cluster is the purple cluster in the upper left. The purple cluster (\#9 ) is quite small and is only distinct from the other press conferences or journalistic video clusters in that it specifically covered PPE topics like mask-wearing.

The blue cluster (\#7 ) in the middle of Figure 3 contains broadcast videos that are at best Covidadjacent. While these contain scant pandemic-related information (and could almost have been dropped from our analysis), they contained basic suggestions like handwashing or vague statements about social distancing. While not brought out in our coding, these videos typically presented celebrities testing positive for COVID-19 and human-interest stories about life in lockdown and empty streets. The black cluster (\#5 contains even more emotional content, also with little in the way of individually actionable information, e.g., stories of hospitals running out of PPE or ventilators.

The cyan cluster (\#6 ) contains both doctors interacting with morning show hosts as well as videos from YouTube channels run by doctors offering reasonably conventional health advice, but, distinct from the red and yellow-green clusters, the videos in this cluster also have some more questionable messages. For example, the March 5th video "Coronavirus Shopping List: What You May Need To Have On Hand," contains common advice about handwashing and self-quarantine, but also features exaggerated language from television $\mathrm{MD}$ and alternative medicine proponent Dr. Oz. Moreover, the video, as its title suggests, has commercial aims (the hosts recommend prominently branded products to buy). The doctor YouTubers in this cluster sometimes present useful information, but this content is also idiosyncratic to the creator, some giving excessive credence to individual studies about hydroxychloroquine, others arguing against lockdowns that "we can't have the cure be worse than the disease". Such discussions are not necessarily misinformation, especially during the early days of the pandemic where less was known definitively, but they were out of step with advice given by public health institutions, and they pander to audiences' more radical impulses, as we note below in observations about video comments.

The green (\#4 ) and magenta (\#10 ) clusters on the bottom of the graph consist of videos addressing both conspiracy theories and less conventional treatments. 5G theories and related homeopathic beliefs about viruses being excreted toxins are the most clearly represented here, though there are other theories as well. Despite the reference to these concepts, many of the videos in the green cluster are actually anti-conspiracy theory videos, for which a characteristic form is to do a round-up of conspiracy theories to ridicule. From the relative absence of videos favoring these ideas (a few came to our attention in data collection), it appears that YouTube has succeeded in removing the most obviously conspiratorial content while leaving anticonspiracy theory content relatively untouched.

In the magenta cluster there is a consistent use of homeopathic, new age, or home-remedy thinking rather than relying upon medical experts. Most of these videos are DIY content instructing viewers in how to make masks, and stock up on food, sometimes encouraging the use of less harmful treatments like consuming large amounts of Vitamin C. While not ideal from a public health standpoint, our sample suggests that such content was not especially prevalent and that YouTube's deletion of more extreme content from these content creators was effective in limiting the influence of such channels to simply their own subscriber base. Notable 
exceptions are the channel Adrian (UCyIhHTLqM1noQbdFuX45EaA) ${ }^{1}$, whose videos argue against Germ Theory, and videos presenting vaccination skepticism even at the early stages of the pandemic.

\subsection{Broader Observations}

In the process of collecting, culling and coding the videos, we made systematic qualitative observations pertaining to pandemic information on YouTube. What follows are those that suggest specific dangers to communicating medical information in this or future health emergencies.

4.2.1 Advertisement as Cover. While our observations lead to the conclusion that YouTube has been reasonably effective at culling obviously misleading information, we did observe a key loophole: advertisements. Video advertisements on the YouTube platform are simply videos flagged as such with the uploading party paying money for the videos to be shown as advertisements. While advertisements are subject to a similar level of scrutiny for the purposes of video deletion, they serve as a means of propagating videos with misinforming content beyond the scope of the recommendation algorithm.

A key example of this is a video (LYRafT5BxCQ) from the channel London Real, which advertised the release of "Plandemic Part 2" on London Real's own website, rather than on YouTube. London Real's owner, Brian Rose, was involved in the production of "Plandemic, Part 1", which promotes a conspiratorial narrative involving pharmaceutical companies, medical doctors, and Dr. Anthony Fauci. The advertisement has since been deleted, possibly due to the time-sensitive nature of the advertisement, or because it was threatened by YouTube. In the same period, London Real's interview of conspiracy theorist David Icke was deleted by YouTube as hate speech, and much of "Plandemic Part 2" contains a similar (or the same) interview between Rose and Icke. While the approach would not work if the content were entirely hosted on YouTube, advertisements allow spreading a message to a YouTube audience while submerging the nature and location of the misinformation.

4.2.2 Covid Hobbyist Audience. Large audiences hungry for news about the pandemic are sometimes dangerous to the integrity of YouTube's content. With people often at home in lockdowns, consumption of online streaming content ballooned, and while many sought conventional entertainment, some became very

1 YouTube links show only the video or channel ID. The hyperlink will take you to the full URL. interested in learning as much as possible about the pandemic, irrespective of whether that information was in any way individually actionable. One channel, DoctorMike (DoctorMike) regularly made the point that constantly consuming information about the pandemic was not useful and that the simple message of hygiene, following lockdowns, and social distancing were all that the general public needed and more information was more likely to stress people than help them. While possibly true, many people did desire more detailed and technical information about what was disrupting their lives.

Official sources predominated the YouTube space, with consistent messaging about hygiene, lockdowns, and social distancing. However, often the only new information in broadcast news or press conferences were morbidity and mortality statistics. Deep dives into the science of the disease and speculation about possible treatments did not merit official attention, especially when the science of the pandemic was unclear and there had been little time for peer-reviewed studies. Where conventional broadcast media would not provide this information, YouTube content creators were available to serve this new "covid hobbyist" audience.

4.2.3 Doctor Channels. As what we call "doctor channels" pivoted to meet the demand of the covid hobbyist audience, their viewership and influence on YouTube rose. These channels are YouTube channels owned and operated by MDs and PhDs in health-related fields; most existed prior to the pandemic, in which they found a heightened relevance. They vary considerably in their foci and approaches, but all possess an unusual degree of authority compared to news broadcasters or other content creators, as they combine expert qualifications with the appearance of authenticity desirable in social media. Some of these channels unambiguously did good work informing the public, but the possession of an MD does not inherently imply agreement with medical or public health consensus. This may lead to misinformation given with the authority of a doctorate.

The YouTube channels MedCram (Medcram) and Dr. John Campbell (Campbellteaching) provide examples of what can happen on the modest end of misinforming content. Both channels existed prior to the pandemic as teaching aids for medical practitioners but changed their content afterwards to focus on detailed medical information about COVID-19. Both did this by attempting to explain medical news and research papers to a broader audience, and both display idiosyncrasies in what subjects they cover, what treatments they favor as promising, and what research they do not believe. 
While this is a normal part of scientific debate, the formats of these videos suggest to the audience that they are listening to experts, and when, as in the case of MedCram, those experts support fringe treatments like "Forest Bathing" or defend hydroxychloroquine long after medical institutions reject it, those medical opinions can become misinformation to the general public.

Szmuda et al. [19] reported that MedCram videos were among the top five best videos on the pandemic on YouTube in the first months of the pandemic, according to their DISCERN scoring system. The channel's stranger videos we noticed appeared outside of that study's very early timescale. Nonetheless, it remains problematic for videos with an authoritative appearance to end up engaging with questionable material. This is not to say that MedCram exclusively spread misinformation, although multiple videos on the channel were at one point deleted by YouTube as misinforming; all were later restored after the channel owner's protest. Dr. John Campbell regularly debated the possible value of hydroxychloroquine, and while he finally denounced its value as a therapeutic in a video published on November 10, 2020, that uncertainty was problematic for his audience, some of whom adopted passionate stances on the subject, as viewed through the comments on his videos.

Science communication was particularly difficult during this period of the pandemic. The widespread use of pre-print publication papers, sometimes motivated by profit or politics, and high-profile retractions of papers made assessing the state of scientific knowledge challenging at best. Hydroxychloroquine in particular was the subject of multiple article retractions at this early stage, both for and against its use. With such confusion, it is understandable that science communicators might adopt neutral postures towards controversy, but when the controversy is greater in political or media spheres than in the scientific consensus, neutrality can encourage misinformation among their viewers.

Some doctor channels intentionally spread unorthodox theories. As the pandemic became increasingly politicized, channels like Dr. Sam Bailey (DrSamBailey) questioned the value of social distancing, leading to the deletion of several videos; in 2021 she also started questioning the germ theory of disease, thereby undermining an important pillar of public health communication in the pandemic. While YouTube provides a platform for institutions to convey their information to the public, its openness and lack of gatekeeping mechanisms permit contradictory messaging from doctors who possess a similar kind of authority to public health institutions in the eyes of the public. This problem needs to be addressed as part of an overall public health communication strategy.
4.2.4 Dissident Doctors. Despite our finding of primarily news-like content, some tendencies of health misinformation were still notable. Homeopaths and naturopaths during the pandemic were somewhat difficult to track, partially because YouTube was already deleting explicit statements that COVID-19 didn't exist, but also because many of the main figures in spreading homeopathic ideas about the pandemic like Dr. Andrew Kaufman or Thomas Cowan did not spread their ideas very much through their own YouTube channels and instead appeared as guests on a wide variety of other channels, giving them a broader reach. While we cannot state the intention behind this approach, this situation enabled these ideas to survive content deletion more effectively than if the content was presented on a single channel.

The exosome theory commonly presented by these figures also reveals some techniques for spreading misinformation. Broadly, this theory proposes that viruses don't exist and that all evidence of them is actually exosomes secreted from cells in response to toxins in the environment, with the substance viewed as causing COVID-19 to vary between proponents, from $5 \mathrm{G}$ towers to microplastics. While this theory is profoundly flawed, research and investment on actual therapeutic applications of exosomes has become increasingly popular over the last two decades [26]. Videos from biomedical companies explaining the basics of exosomes with well-made visualizations have been repurposed (presumably without permission) by homeopaths and naturopaths to add production value to their own presentations.

Another kind of dissent was exhibited by the "Bakersfield Doctors" Erickson and Massihi, who first appeared on a Bakersfield, CA ABC affiliate morning program, a clip of which was uploaded to YouTube on April 26 and widely shared. This appearance also precipitated later interviews and appearances on Fox News as well as other YouTube channels (for Erickson, in particular). Alongside Plandemic, which appeared around the same time, this video got considerable response for its anti-lockdown views supported by the authority of the two urgent care MDs. Both the Bakersfield doctors videos and Plandemic were regularly deleted by YouTube, but the videos were reuploaded to unrelated channels. YouTuber doctors were predominantly negative in their assessment of these arguments, even when they shared this antilockdown stance (7aT-Nb0nDeY) because of their dubious medical claims and non-rigorous use of data.

4.2.5 Singaporean Experience. While our study primarily included videos in English, this did provide some examples of English-language COVID-19 content from outside the US and Commonwealth, most 
prevalently from CNA (channelnewsasia), a Singaporean state-run media channel. Unlike the more panicked media coverage from the US, CNA's reporting very quickly (in mid-January) described the mitigation techniques of social distancing and proper hygiene and their media figures reacted with greater awareness of how to effectively cover a disease outbreak. This is perhaps explained by Singapore's regular experience with disease outbreaks and suggests to us that part of the problems with media coverage of the pandemic are at least partially a matter of a lack of experience with covering such stories.

\section{Conclusions}

The findings of this study may be considered in three parts: methodology, confirmation of previous work, and new observations about COVID-19 information on YouTube.

Methodologically, the present study contributes an approach to topics requiring more complex codings than simple categories. Here, we employed a method of coding statements using semantic slots for which we developed categories, hence benefitting from the concepts and rigors of grounded-theory content analysis. The approach scales to some extent, although the complexity of the codes and the messages they are coding requires greater time commitment. Nonetheless, the main problem for our study is the need to watch and observe many videos, something for which there is no real analytical substitute. While automated coding promises increased scale, and the slot system suggests an information extraction approach, the available resources are inadequate to this task. Transcriptions are not available for every video, and even when they are the quality is poor as the system is not updated for fastmoving topics ("COVID" was rendered variously, including, e.g., as "kovat"). Furthermore, any approach attempting to achieve scale automatically will have to squarely confront declining access to API quotas.

Beyond this, there are things we would want to improve when undertaking new studies. More consideration needs to go into the coding categories if counter-messages and misinforming arguments are to be reflected clearly. These also are often structured within a video as sequences of arguments which might, e.g., go from vaccine skepticism to a conclusion of full virus denial, as was the central argument of Plandemic. Our method does not yet address these issues, or how they contribute to the kind of misinformation a specific message represents. Additional refinement might simplify the coding scheme: many of our codes were infrequently used, and the full set of statements was large and difficult to handle analytically. However, the coding is difficult to simplify, given the complex nature of pandemic misinformation, which is also situated in a complex, contested context of medical, economic, and political ideologies. The novel, evolving, dynamic subject matter is inescapably challenging.

We underscore the importance and salience of broadcast news with respect to the COVID-19 pandemic [20]; the role of these will need to be considered for other health information topics. Television doctors like Dr. Oz often communicate messages similar to concurrent misinforming or problematic messages found on YouTube. Studies would also benefit from incorporating additional nuance into the study of COVID-19. Assessing information quality in this case is difficult, as the best guidance from public health authorities changed greatly, more than once, between January and May, so minimally what counts as misinformation depends closely on context.

Additionally, the particular qualities of YouTube as a medium need to be incorporated into quality metrics like DISCERN, alongside adaptations for the peculiarities of a novel virus. DISCERN was originally designed to assess communication about known ailments and their treatment, so that patients and doctors could make appropriate treatment decisions. One of its advertised advantages is that users do not need specialized knowledge about the medical issue at hand; the tool does not address the scientific soundness of supporting publications. While perhaps reasonable in intent, during a novel pandemic, medical knowledge is necessarily evolving and incomplete, meaning it is easier to give unsubstantiated claims the appearance of scientific substantiation. Further, public health efforts require behavior changes that are more complex, prone to rapid change, and not necessarily about individual treatments so much as broad mitigation strategies which characteristically need to be oversimplified. While Szmuda et al. [19] produced their work rapidly at a time when scholarly approaches were rare and badly needed, they neglected to adapt DISCERN to the idiosyncrasies of YouTube, as was done in Loeb et al. [18] or to the different conditions of a pandemic. This suggests a need for much future work to develop medical media assessment techniques to specific social media platforms, genres of communication, etc.

Finally, we stress that in the YouTube environment in particular, it is important to recognize the various covert paths through which misinformation is reinforced. YouTube's environment is complex, enabling viewers to pass rapidly among competing, even adversarial presenters and messages. This is as true for medical and health information as it is for commercial and political messaging: alternative medical practitioners with stigmatized medical theories seize upon the uncertainties of a novel pandemic to pursue their agendas. These purposes can run counter to the prevailing concerns of public health by promoting non-credible theories (e.g. "terrain theory" vs. "germ 
theory", waste products vs. viruses, misapplication of exosome theory, etc.), thereby confounding and complicating public health messaging. Where other studies might point to state propaganda, failures in public health messaging and politicization as contributing factors, our observations suggest a key role for the opportunistic exercise of pre-existing medicinal counter-ideologies in the development of the infodemic.

\section{References}

[1] WHO. WHO Situation Report 13. Feb 2, 2020. https://www.who.int/docs/defaultsource/coronaviruse/situation-reports/20200202-sitrep13-ncov-v3.pdf?sfvrsn=195f4010 6.

[2] M. Cinelli, W. Quattrociocchi, A. Galeazzi, C.M. Valensise, E. Brugnoli, A.L. Schmidt, P. Zola, F. Zollo and A. Scala. "The covid-19 social media infodemic." Scientific Reports, 10(1), 2020. Pp.1-10.

[3] D. Allington, B. Duffy, S. Wessely, N. Dhavan, and J. Rubin. "Health-Protective Behaviour, Social Media Usage, and Conspiracy Belief during the COVID-19 Public Health Emergency." Psychological Medicine. 2021.

[4] H. Horsburgh and D. Barron. "Who are the experts? Examining the promotion of misleading and harmful nutritional information." pp. 100-115. In Lavorgna, A., \& Di Ronco, A. (Eds.). Medical Misinformation and Social Harm in Non-Science Based Health Practices: A Multidisciplinary Perspective. Routledge. New York. 2019.

[5] X. Cheng, C. Dale and J. Liu, "Statistics and social network of youtube videos." In: IWQoS 2008, 16th International Workshop on Quality of Service. IEEE Computer Society, Los Alamitos, CA, 2008. pp. 229-238.

[6] S. Cunningham, D. Craig, and J. Silver. YouTube, multichannel networks and the accelerated evolution of the new screen ecology. Convergence, 22(4), 2016. pp. 376391.

[7] J. Paolillo, "Structure and network in the YouTube core", In: Proceedings of the 41st Annual Hawaii International Conference on System Sciences, Los Alamitos, CA, 2008.

[8] J. Klausen, E.T. Barbieri, A. Reichlin-Melnick and A.Y. Zelin. "The YouTube Jihadists: A social network analysis of Al-Muhajiroun's propaganda campaign." Perspectives on Terrorism, 6(1), 2012. Pp.36-53.

[9] J. Paolillo, S. Ghule, and B.P. Harper. A Network View of Social Media Platform History: Social Structure, Dynamics and Content on YouTube. In Proceedings of the 52nd Hawaii International Conference on System Sciences. 2019.

[10] Burgess, J., and Green, J., YouTube: Online video and participatory culture. John Wiley \& Sons. 2018.

[11] J. Kim, "The institutionalization of YouTube: From usergenerated content to professionally generated content", Media, Culture \& Society, 34(1), 2012. Pp. 53-67.

[12] G. Veletsianos, R. Kimmons, R. Larsen, T.A. Dousay and P.R. Lowenthal. Public comment sentiment on educational videos: Understanding the effects of presenter gender, video format, threading, and moderation on YouTube TED talk comments. PloS one, 13(6), 2018.

[13] M.H. Ribeiro, R. Ottoni, R. West, A. F. Virgílio Almeida, and W. Meira. "Auditing Radicalization Pathways on YouTube." 2019.

[14] M. Ledwich, and A. Zaitsev. "Algorithmic Extremism: Examining YouTube's Rabbit Hole of Radicalization." 2019.

[15] M. Faddoul, G. Chaslot and H. Farid. "A Longitudinal Analysis of YouTube's Promotion of Conspiracy Videos." arXiv preprint arXiv:2003.03318. 2020.

[16] R. Briones, X. Nan, K. Madden, and L. Waks. "When Vaccines Go Viral: An Analysis of HPV Vaccine Coverage on YouTube." Health Communication 27 (5), 2021. pp. 478-85.

[17] S. Ekram, K.E. Debiec, M.A. Pumper, and M.A. Moreno. "Content and Commentary: HPV Vaccine and YouTube." Journal of Pediatric and Adolescent Gynecology 32 (2), 2019. pp. 153-57.

[18] S. Loeb, S. Sengupta, M. Butaney, J. N. Macaluso Jr., S. W. Czarniecki, R. Robbins, R.S. Braithwaite, L. Gao, N. Byrne, D. Walter and A. Langford. Dissemination of misinformative and biased information about prostate cancer on YouTube. European Urology, 75(4), 2019. 564-567.

[19] T. Szmuda, M.T. Syed, A. Singh, A. Shan, C. Özdemir, and P. Słoniewski. "YouTube as a Source of Patient Information for Coronavirus Disease (COVID-19): A Content-Quality and Audience Engagement Analysis." Reviews in Medical Virology 30 (5).

[20] N. Marchal, and H. Au. "Coronavirus EXPLAINED": YouTube, COVID-19, and the Socio-Technical Mediation of Expertise. Social Media+ Society, 6(3), 2020.

[21] L. Scrucca, M. Fop, T.B. Murphy and A.E. Raftery. "mclust 5: clustering, classification and density estimation using Gaussian finite mixture models." The R Journal 8(1), 2016. pp. 289-317.

[22] Jackson, J.E. A user's guide to principal components (Vol. 587). John Wiley \& Sons. 2005.

[23] J. Chiquet, M. Mariadassou and S. Robin. Variational inference for probabilistic Poisson PCA. Annals of Applied Statistics, 12(4), 2018. pp. 2674-2698.

[24] R Core Team, "R: A language and environment for statistical computing", R Foundation for Statistical Computing, Vienna, Austria.

[25] C. Fraley, and A. E. Raftery. MCLUST: Software for model-based cluster analysis. Journal of classification, 16(2), 1999. pp. 297-306.

[26] B. Wang, D. Xing, Y. Zhu, S. Dong and B. Zhao, "The state of exosomes research: a global visualized analysis." BioMed Research International, 2019. 